\title{
Gesundheitsmediation: ernsthafter Dialog mit Patientinnen und Patienten
}

Jürg Gasche *

* Der Autor ist Mediator und Rechtsanwalt in Zürich.

Korrespondenz: Jürg Gasche M.A. Mediator SDM/M.B.L. - HSG Rechtsanwalt Strassburgstrasse 10 CH-8004 Zürich info@construmediat.ch

\section{Einleitung}

Moderne Patientinnen und Patienten sind nicht geduldig abwartend und mit allem zufrieden, was ihnen von der Ärztin, vom Arzt zukommt. Die Beziehung zwischen Arzt und Patient hat sich mit der gesellschaftlichen Entwicklung der vergangenen Jahrzehnte stark verändert. 1994 wurden durch die WHO und seither auch in kantonalen Gesetzen Patientenrechte definiert und zudem Instanzen geschaffen, an die sich Unzufriedene mit ihren Reklamationen wenden können. Um über diese Entwicklungen zu informieren und um Erfahrungen auszutauschen und zu diskutieren, führten das «Institut du droit de la santé» (IDS) und das «Centre de recherche sur les modes amiables et juridictionnels de gestion des conflits» an der Universität Neuenburg ein Kolloquium mit internationaler Beteiligung durch. Präsentiert wurden Erfahrungen aus Italien, Belgien und der Suisse romande. Danach rundeten grundsätzliche Reflexionen zur Beziehung von Arzt und Patient und zur Gesundheitsmediation sowie eine Schlussdiskussion die Tagung $\mathrm{ab}$.

In fünf von sechs Kantonen der französischen Schweiz können sich unzufriedene Patienten an spezielle Stellen für Gesundheitsmediation wenden. Nur in Neuenburg fehlt bisher eine solche Regelung. Die Kantone Bern und Tessin wurden ebenfalls in die Untersuchung einbezogen. Bern hat seit 2005 eine Regelung, im Kanton Tessin gibt es nichts Derartiges. Die Juristin Johanne Gagnebin untersuchte die verschiedenen Modelle der Gesundheitsmediation. Die Chance der Mediation im Gesundheitswesen sieht sie im ernsthaften Dialog mit den Patientinnen und Patienten sowie in der besseren Wahrung ihrer Würde.

\section{Waadt}

Dr. Chantal Thouverez ist seit 2004 «médiatrice cantonale de la santé de l'Etat de Vaud» und behandelte seither 127 Fälle. Im Jahr 2004 waren es 30, und jedes Jahr wuchs die Zahl um mehr als 20 Prozent. Die Erfahrungen sind positiv, denn ihre Mediationen führten zu Verbesserungen von Leistungen in Gesundheitseinrichtungen

\section{Médiation et santé}

Les patients modernes ne sont plus tellement patients, passifs et content avec tout ce qu'ils reçoivent du coté de leur médecin. Les relations entre médecins et patients ont changés en parallèle avec les développements dans la société civile. En 1994 la OMS a statuée les droits des patients et des lois de plusieurs cantons suisses romandes de même. Ces derniers ont crées des instances auxquels les patients désabusés peuvent adresser leurs réclamations. Afin d'informer de ces développements, d'échanger des expériences et de les discuter, I'Institut du droit de la santé (IDS) en commun avec le Centre de recherche sur les modes amiables et juridictionnels de gestion des conflits ont organisé au sein de I'Université de Neuchâtel un colloque avec une participation internationale. Des expériences italiennes, belges et suisses ont été présentées.

und in etlichen Fällen auch in den Beziehungen zwischen den Patientinnen, Patienten und Fachpersonen. Vor den gemeinsamen Mediationssitzungen führt sie mit beiden Seiten ein Vorgespräch. Die Mediationen wirken konstruktiv und fördern die Verantwortlichkeit für das jeweilige eigene Handeln, sie sind wirksam, schnell, diskret und haben einen pädagogischen Effekt: Sie führen $\mathrm{zu}$ Verhaltensänderungen und $\mathrm{zu}$ Organisationsentwicklungen. In den Mediationen werden alle vorgebrachten Probleme behandelt mit Ausnahme von Honorarfragen, Berufsfehlern, sexuellem Missbrauch, Diebstahl und anderen Vermögensdelikte sowie Versicherungsstreitigkeiten. In solchen Fällen werden die betroffenen Personen an die zuständige Stelle weitergeleitet.

Die Fachpersonen aus dem Gesundheitsbereich kommen gerne zu den Mediationen, um einen Konflikt mit einem Patienten, einer Patien- 
tin vertraulich zu bearbeiten, ohne dass Vorgesetzte informiert werden.

Für Konflikte mit Ärztinnen und Ärzten besteht die Möglichkeit, sich wahlweise an die kantonale Mediatorin oder an die Ärztegesellschaft zu wenden.

\section{Freiburg i.ü.}

José-Manuel Valentin berichtete, dass im Kanton Freiburg die Aufsichtskommission über die Gesundheitsfachleute und die Patientenrechte seit 2001 lediglich 6-8 Fälle pro Jahr zu behandeln hatte. Mehr als die Hälfte davon aus dem Bereich der Psychiatrie. Die vorgebrachten Rügen betrafen die Hospitalisierung, Informationsdefizite, medizinische Fehler, Finanzfragen, Zwangsmassnahmen, Begehren für Wiedergutmachungen in Form von Entschuldigungen oder Geldzahlungen. Bei Kunstfehlern führt die Klärung über eine Expertise ausserhalb der Mediation. Schadenersatzbegehren werden auf den Zivilprozessweg gewiesen. Die Zielsetzung bei der Behandlung der Fälle ist es, den Dialog zwischen Patient, Patientin und Fachperson wiederherzustellen und den Konflikt zu bewältigen.

\section{Toskana}

Manuele Bellonzi, Lehrbeauftragter der Universität Pisa, berichtete, wie die Patientinnen und Patienten in der Toskana ihre Feststellungen, Einsprüche, Anzeigen und Klagen ausserhalb des konventionellen Rechtsweges bei den dafür eingerichteten gemischten Vermittlungskommissionen vorbringen können. Diese Kommissionen behandelten in den Jahren 2003 (77), 2004 (85) und 2005 (159) insgesamt 321 Fälle. Die betroffenen Patientinnen und Patienten können zur Behandlung ihrer Eingabe eingeladen werden. In den Verfahren, die zu Beginn vorwiegend schriftlich geführt wurden, wird heute mehr mit den Beteiligten gesprochen. Es hat sich gezeigt, dass nebst der ursprünglich beabsichtigten strukturierten Rückmeldung zur Verbesserung der Qualität der Gesundheitseinrichtungen ein Bedarf nach Wiederherstellung gestörter Vertrauensbeziehungen besteht.

\section{Belgien}

Die Sozialpsychologin Elisabeth Volkrick, Professorin der Universität Leuven, führt die Verbreitung der Mediation zur Bearbeitung von Konflikten auf tiefgreifende Änderungen in der Gesellschaft zurück. Zusammenleben und Zusammenarbeit haben sich verändert, die Vielfalt von Sprachen und Kulturen erhöht die gesellschaftliche Komplexität. Oft gibt es keine von allen Beteiligten anerkannte Norm, denn es be- gegnen sich mehrere Normenwelten. Die Frage lautet: «Was ist für Sie richtig?»

Mangels einheitlicher Normvorstellungen leiden sowohl staatliche wie private Institutionen unter einem Verlust an Legitimierung und Vertrauen. Da ist die Mediation eine Art Neulegitimierung von unten. In Belgien begann nach grossen Strassendemonstrationen und Massenprotesten gegen Missstände im Gesundheitswesen ein Umdenken. Im Jahr 2002 wurde das Gesetz über die Patientenrechte erlassen. Die darin vorgesehene Mediation ist allerdings noch nicht wirklich etabliert, denn das Gesetz lässt viele Fragen offen: Weder die Mediation noch die Kompetenzen des Mediators, seine Unabhängigkeit von den betroffenen Institutionen, die Vertraulichkeit des Verfahrens und das Berufsgeheimnis sind definiert. So kommt es vor, dass der vom Spitaldirektor ernannte Mediator diesem seine Fälle referiert.

Die Mediatoren haben sich nun zum Erfahrungsaustausch organisiert, und die Mediation im Gesundheitswesen wird ohne Zweifel weiter entwickelt werden. Nach den bisherigen Erfahrungen genügt die Reduktion auf die Erledigung der Beschwerden (efficacité) nicht. Es wird darum gehen, gestörte Beziehungen zwischen Patientinnen, Patienten und Medizinfachleuten in einer Weise zu bearbeiten, welche die Selbstreflexion in den Gesundheitsorganisationen anregt. Ziel ist es, dass die Fachleute die Patientinnen und Patienten besser informieren, ihnen besser zuhören, die Beziehung zum Patienten interaktiv verstehen und mit ihm Konsens herstellen (légitimité).

\section{Veränderung der Beziehung zwischen Ärztinnen/Ärzten und Patientinnen/Patienten}

Der Soziologe Dr. Raphaël Hammer, Lehrbeauftragter der Universität Genf, sieht die modernen Patientinnen und Patienten als gut informiert, fordernd, skeptisch und gegenüber den Ärztinnen und Ärzten oft in der Haltung von Kunden gegenüber Dienstleistenden. Die Vertrauensbeziehung zwischen Ärztinnen, Ärzten, Patientinnen und Patienten beschreibt er mit fünf Typologien: vom klerikalen Vertrauen mit braven und folgsamen Patienten, die weder Arbeit noch Urteil des Arztes hinterfragen, bis hin zum rationellen Vertrauen, wo der Arzt bescheiden und sich seiner Grenzen bewusst ist und so von den Patienten als glaubwürdig wahrgenommen wird. Die Ärztinnen und Ärzte mit der besten kommunikativen Beziehung zu gut informierten Patientinnen und Patienten haben am wenigsten Klagen zu befürchten. 